\title{
Interposición de colon transverso como alternativa de reconstrucción tras la esofagogastrectomía total*
}

\author{
Drs. RODRIGO ABULARACH C. ${ }^{1}$, FRANCISCO VENTURELLI M. ${ }^{2}$, \\ RICARDO CERDA C. ${ }^{1,2}$, ALVARO URIZAR G. ${ }^{1}$, EDUARDO LIRA E. ${ }^{1}$, \\ YAMILE HAITO CH. ${ }^{2}$, CARLOS LOYOLA R. ${ }^{2}$, CARLOS MATUS F. ${ }^{1}$ \\ 1 Servicio de cirugía Hospital Barros Luco Trudeau. \\ 2 Servicio y Departamento de Cirugía Hospital Barros Luco Trudeau. Campus Sur. Facultad de Medicina, \\ Universidad de Chile. \\ Santiago, Chile.
}

Transverse colon interposition as an alternative to reconstruction after total esophagogastrectomy

\section{Introducción}

Existen múltiples indicaciones de esofagogastrectomía tanto para patología maligna como para benigna, siendo la más frecuente en la primera el cáncer de la unión gastroesofágico (UGE) con compromiso extenso del esófago abdominal y torácico inferior (Siewert tipo IV según Burmeister) y en la segunda, la lesión gastroesofágica por cáusticos ${ }^{1-3}$.

En cualquiera de estos casos, el ascenso de colon constituye una indicación de necesidad para la reconstitución del tránsito esofágico, el cual puede ser ascendido por vía retroesternal, por mediastino posterior, subcutáneo o transpleural ${ }^{4-6}$.

Las alternativas de coloplastía para realizar la reconstitución del tránsito tras la esofagogastrectomía son ${ }^{5,7-10}$.

1. Ileocoloplastía derecha isoperistáltica

2. Coloplastía derecha isoperistáltica

3. Coloplastía transversa isoperistáltica

4. Coloplastía transversa anisoperistáltica

5. Coloplastía izquierda anisoperistáltica.
En el caso de la ileocoloplastía y coloplastía derecha isoperistáltica, la irrigación dependerá de los vasos cólicos derechos superiores, en el caso de la coloplastía transversa isoperistáltica el pedículo depende de los vasos cólicos izquierdos superiores, y en la coloplastía transversa anisoperistáltica su pedículo depende de los vasos cólicos medios.

Desde el punto de vista técnico la interposición de colon derecho exige una menor disección en relación a la movilización y al ascenso del mismo, sin embargo, en algunas ocasiones no se cumplen las condiciones ideales para el ascenso de este, teniendo que recurrir a otro tipo de injerto por lo que es importante el conocer la anatomía y técnica quirúrgica para realizar otros tipo de coloplastía.

El objetivo de este estudio es la descripción de la técnica quirúrgica de la coloplastía transversa como reemplazo esofágico tras la esofagogastrectomía total por cáncer de UCE con extenso compromiso del esófago abdominal y torácico inferior.

*Recibido el 2 de mayo de 2011 y aceptado para publicación el 3 de junio de 2011.

Correspondencia: Rodrigo Abularach C.

Gran Avenida 3204, Santiago, Chile.

roabularach@hotmail.com 


\section{Técnica quirúrgica}

A todos los pacientes se les realiza estudio de colon preoperatorio mediante una colonoscopia larga y de resultar esta normal se prepara el colon 2 días antes de la cirugía con dieta, antibióticos, fleet enema y enema evacuante.

Terminada la resección del esófago y el estómago por doble abordaje abdominal y cervical, se procede a realizar el primer tiempo de la coloplastía transversa que consiste en la movilización completa del colon derecho, del colon transverso y del ángulo cólico izquierdo, teniendo el cuidado de no lesionar o estirar los vasos cólicos izquierdos superiores y no comprimir la vena mesentérica inferior (primer tiempo crítico). El mesenterio del ángulo cólico izquierdo debe ser movilizado hasta la aorta, no siendo necesaria la movilización de la unión sigmoidoilíaca y sigmoides.

La disección de los vasos cólicos medios y derechos superiores deben realizarse a nivel de su nacimiento en relación a los vasos mesentéricos superiores. Se debe respetar a toda costa la arcada vascular marginal del injerto en su totalidad (segundo tiempo crítico). Este tipo de coloplastía se encontrará entonces pediculada por los vasos cólicos izquierdos superiores (Figura 1).

Terminada la disección realizamos una prueba de

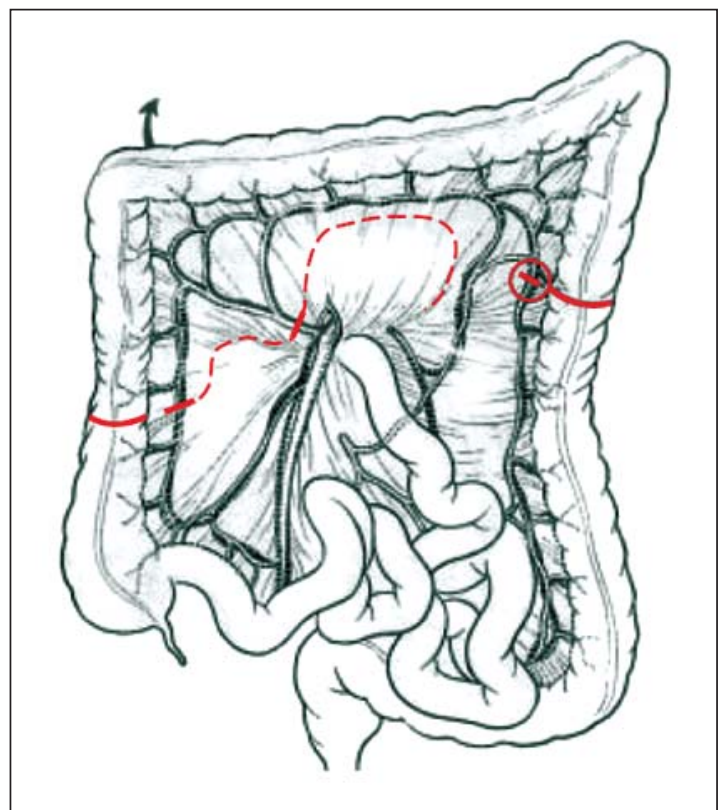

Figura 1. Coloplastía transversa isoperistáltica ${ }^{20}$. En el círculo rojo, la arcada marginal del lado izquierdo debe ser respetada. clampeo sobre los vasos cólicos medios y derechos (un bulldog arterial y un bulldog venoso).

Esta prueba de clampeo autoriza la coloplastía en las siguientes circunstancias:

Presencia de pulso arterial en el segmento de colon ascendente que debe llevarse al cuello; ausencia de estasis venosa en esta zona. Superada la prueba del clampeo se procede a realizar la ligadura vascular a ras de los vasos mesentéricos superiores siendo indispensable no interrumpir la colateralidad en relación a la arcada marginal.

La longitud efectiva del segmento que ascenderá al cuello se encuentra generalmente a media altura del colon ascendente. A continuación procedemos a seccionar este segmento con sutura mecánica (lineal cortante), posteriormente seccionamos el colon distal próximo al ángulo esplénico del colon o algunos centímetros por debajo del mismo (colon descendente) decisión que se tomará de acuerdo al largo necesario de colon para realizar un ascenso sin tensión. En caso de que la prueba de clampeo no autorice la coloplastía transversa isoperistáltica se procede a clampear los vasos cólicos izquierdos a ras de los vasos mesentéricos inferiores para proceder posteriormente a seccionarlos y realizar la coloplastía transversa anisoperistáltica pediculada por los vasos cólicos medios (Figura 2).

Terminada la preparación del injerto la etapa

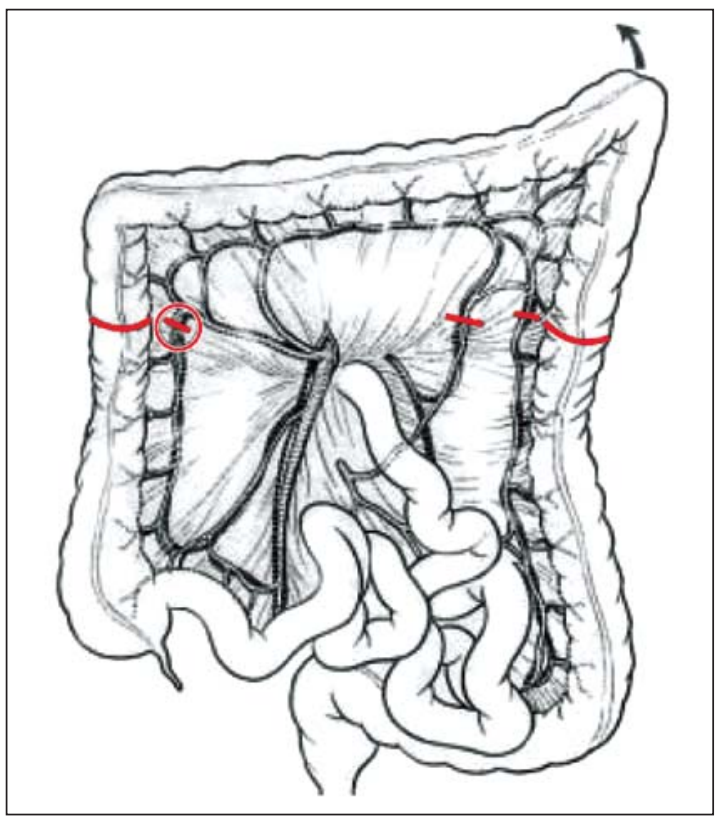

Figura 2. Coloplastía transversa anisoperistáltica ${ }^{20}$. En el círculo rojo, la arcada marginal del lado derecho debe ser respetada. 


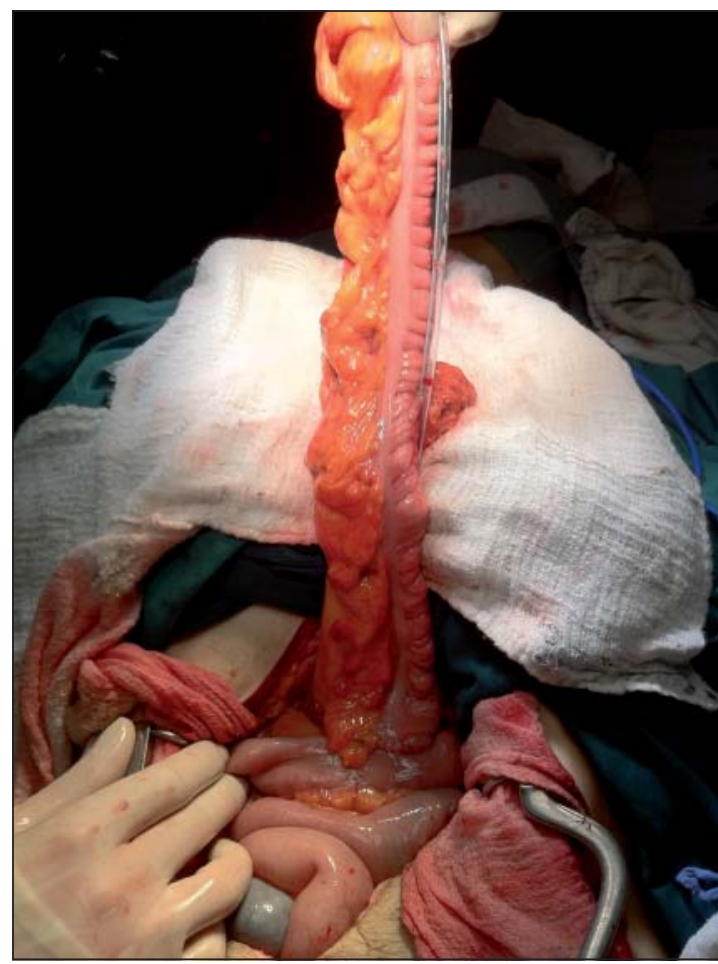

Figura 3. Injerto de colon transverso pediculado isoperistáltico con anastomosis coloyeyunal.

de la reconstrucción sigue de la siguiente manera (Figuras 3 y 4):

1. Colo-coloanastomosis termino-terminal en dos planos manual con monocril 3-0 y cierre de brecha mesocolónica con seda 3-0.

2. Confección del asa en Y de roux de $30 \times 70 \mathrm{~cm}$. manual con monocril 3-0 monoplano punto continuo.

3. Colo-yeyuno anastomosis T-L antecólica manual con monocril 3-0 monoplano continuo.

4. Ascenso de coloplastía isoperistáltica por mediastino posterior hasta la región cervical, la cual debe llegar como primicia; sin tensión, tracción y compresión del pedículo vascular principal y marginal.

5. Esófago-coloanastomosis T-L manual con monocril 3-0 monoplano.

\section{Discusión}

Las alternativas de coloplastía para realizar la reconstitución del tránsito tras la esofagogastrectomía son la: Ileocoloplastía derecha isoperistáltica, Colo-

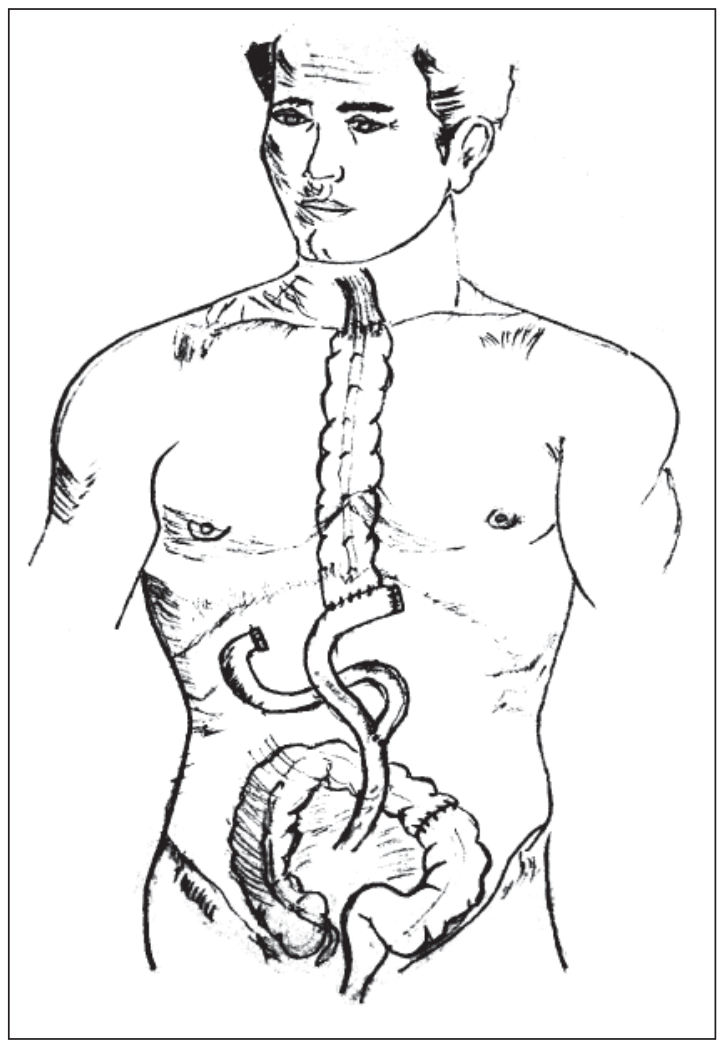

Figura 4. Reconstitución del tránsito esofágico con interposición de colon.

plastía derecha isoperistáltica, Coloplastía transversa isoperistáltica, Coloplastía transversa anisoperistáltica, Coloplastía izquierda anisoperistáltica.

De las opciones antes mencionadas la que tiene más popularidad entre los cirujanos es la coloplastía derecha, ya que requiere una movilización del mismo menos agresiva, con la ventaja de ser isoperistáltica por necesidad manteniendo en forma teórica una mejor función de deglución ${ }^{11-15}$.

Sin embargo, este injerto tiene los siguientes inconvenientes, en relación a su circulación y a su diámetro. En relación al primer punto, la circulación del ciego, la cual deriva de los vasos ileocolocecoapendiculares no es siempre constante y su colateralidad depende en su mayor porcentaje de la arteria y vena ileocoloapendicular (la cual debe ser seccionada) y no así de la arcada marginal ${ }^{9,16}$. Además en un $20 \%$ la cólica derecha está ausente y en un 5\% no existe una anastomosis entre la cólica derecha y la ileocólica, lo cual hace que la coloplastía derecha tenga más riesgo por lo menos teórico de isquemia. En cuanto al segundo punto no siempre existe un diámetro anteroposterior adecuado entre el colon derecho, el desfiladero cervicomediastínico 
y el mediastino posterior, lo que no permite muchas veces un ascenso sin tracción y posterior compresión del injerto, a esto se suma la posible formación de bucles, factores que pueden provocar en mayor o menor grado la isquemia del injerto ascendido, y lo que en definitiva puede derivar en complicaciones que van desde las filtraciones, estenosis hasta necrosis del injerto ${ }^{17}$.

Debido a que se ha visto que el colon ascendido tiene actividad peristáltica, el realizar el injerto de forma isoperistáltico, lograría tener una mejor función de deglución, sin embargo, cuando la prueba del clampeo de los vasos cólicos izquierdos no es satisfactoria se opta por la coloplastía anisoperistáltica pediculada en los vasos cólicos medios ${ }^{11-14}$.

Comenzamos con esta técnica en el Hospital Barros Luco Trudeau el año 2008 como alternativa de reconstrucción tras la esófago gastrectomía total por cáncer. Hasta la fecha el autor ha intervenido 5 esofagogastrectomías totales agregando en uno de los casos una resección pancreática córporo caudal más esplenectomía y hepatectomía lateral izquierda (segmentos II-III) por extensión local del tumor, todos fueron realizados por doble abordaje (abdominal-cervical) y con interposición de colon transverso vía mediastino posterior (4 isoperistálticas y 1 anisoperistáltico). La media de edad fue 50 años (34-68), todos de sexo masculino. La complicación más frecuente fue el neumotórax, el cual se presentó en 4 pacientes, de estos sólo 2 requirieron instalación de pleurostomía. La segunda complicación más frecuente fue la neumonía intrahospitalaria, que se presentó en 3 pacientes, todos respondieron satisfactoriamente al esquema antibiótico. También 2 pacientes cursaron con disfonía transitoria. Hubo 1 sólo caso de fistula de la anastomosis, la cual fue de la anastomosis colocolónica, que se manejo en forma conservadora. No hubo ninguna necrosis del injerto ascendido. La media de días de hospitalización posoperatoria fue de 20 (15-23). En un paciente se practicó el ascenso de colon anisoperistáltico, debido a que la prueba de clampeo no fue satisfactoria.

Al practicar la coloplastía transversa, en especial la isoperistáltica, existe el temor de que se produzca la isquemia del injerto, ya que el pedículo izquierdo es considerablemente de menor calibre que los vasos cólicos medios y derechos. Sin embargo, según varios autores, la probabilidad de que esto ocurra es de un $0-10 \%$, similar a las otras coloplastías ${ }^{16,18,19}$. Es más, un grupo alemán de alta experiencia en reconstrucción del tránsito esofágico muestra $0 \%$ de necrosis del injerto ascendiendo de colon derecho en base al pedículo de la arteria cólica izquierda ${ }^{9,16}$.

Por otra parte, no hay reportes en la literatura que muestren que el ascender este segmento de colon se asocia a una mayor, menor o igual incidencia de dilatación del injerto, estenosis y fistulas de la anastomosis. Por otra parte, para algunos autores la coloplastía transversa a expensas de la cólica izquierda es la técnica de elección para la reconstrucción del tránsito esofágico con colon ${ }^{17}$. Creemos que si bien para muchos cirujanos la coloplastía transversa (iso o anisoperistáltica) no es utilizada como primera elección en la reconstitución del tránsito esofágico, debe ser considerada como una buena alternativa ante el fracaso de otro tipo de coloplastías.

\section{Referencias}

1. Angel A, Burmeister R, Benavides C. Cáncer gastroesofágico. Rev Colomb Cir. 2000;15: 238-42.

2. Braghetto I, Csendes A, Amat J, Parada M, Schutte H, Lizana C, y cols. Reconstrucción del tránsito esofágico con interposición de colon. Rev Chil Cir. 1994; 46:47784.

3. Isolauri J. Colonic interposition for benign esophageal disease. Long-term clinical and endoscopic results. Am J Surg. 1988;155:498-502.

4. Davydov M, Stilidi I, Bokhyan V. Intrapleural colon interposition in gastric carcinoma patients. Eur J Cardiothorac Surg. 2001;20:1063-4.

5. Motoyama S, Kitamura M, Saito R, Maruyama K, Sato Y, Hayashi K, et al. Surgical outcome of colon interposition by the posterior mediastinal route for thoracic esophageal cancer. Ann Thorac Surg. 2007;83:1273-8.

6. Khan AZ, Nikolopolous I, Botha AJ, Mason RC. Substernal long segment left colon interposition for oesophageal replacement. Surgeon 2008;6:54-6.

7. Cheng BC, Xia J, Shao K, Mao ZF, Huang J, Wang TS. Surgical treatment for upper or middle esophageal carcinoma occurring after gastrectomy: a study of 52 cases. Dis Esophagus 2005;18:239-45.

8. Klink CD, Binnebösel M, Schneider M, Ophoff K, Schumpelick V, Jansen M. Operative outcome of colon interposition in the treatment of esophageal cancer: a 20-year experience. Surgery 2010;147:491-6.

9. Fürst H, Hüttl TP, Löhe F, Schildberg FW. German experience with colon interposition grafting as an esophageal substitute. Dis Esophagus 2001;14:131-4.

10. Sakamoto T, Fujimaki M, Tazawa K. Ileocolon interposition as a substitute stomach after total or proximal gastrectomy. Ann Surg. 1997;226:139-45.

11. Paris E. The colon as oesophageal substitute in non-malignant disease Long-term clinical results and functional studies. Eur J Cardio-thorac Surg. 1991;5: 474-8.

12. Stone MM, Fonkalsrud EW, Mahour GH, Weitzman JJ, Takiff H. Esophageal replacement with colon interposition in children. Ann Surg. 1986;203:346-51.

13. Dreuw B, Fass J, Titkova S, Anurov M, Polivoda M, Ottinger AP, et al. Colon interposition for esophageal re- 
placement: isoperistaltic or antiperistaltic? Experimental results. Ann Thorac Surg. 2001;71:303-8.

14. Myers JC, Mathew G, Watson DI, Jamieson GG. Peristalsis in an interposed colonic segment immediately following total oesophagogastrectomy. Aust N Z J Surg. 1998;68:278-80.

15. Kao CH, Wang SJ, Chen CY, Chen CL, Yeh SH. The motility of interposition in patients with esophageal carcinoma after reconstructive esophageal surgery. Clin Nucl Med. 1993;18:782-5.

16. Fürst H, Hartl WH, Löhe F, Schildberg FW. Colon interposition for esophageal replacement: an alternative technique based on the use of the right colon. Ann Surg. 2000;231:173-8.
17. Maish MS, DeMeester SR. Indications and technique of colon and jejunal interpositions for esophageal disease. Surg Clin North Am. 2005;85:505-14.

18. Mine S, Udagawa H, Tsutsumi K, Kinoshita Y, Ueno M, Ehara K, et al. Colon interposition after esophagectomy with extended lymphadenectomy for esophageal cancer. Ann Thorac Surg. 2009;88:1647-53.

19. Cerfolio RJ, Allen MS, Deschamps C, Trastek VF, Pairolero PC. Esophageal replacement by colon interposition. Ann Thorac Surg. 1995;59:1382-4.

20. Sauvanet A, Belghiti J. Cirugía del cáncer de esófago en: encycolpedie medico-chirurgicale, técnicas quirúrgicas aparato digestivo. París: Editorial Rue Linois, 2002;E-40-195:1-34. 